\title{
Missed opportunity? Worsening breathlessness as a harbinger of death: a cohort study
}

\author{
David C. Currow ${ }^{1,2}$, Joanna M. Smith ${ }^{3}$, Phichai Chansriwong ${ }^{4}$, \\ Simon I.R. Noble ${ }^{5}$, Theodora Nikolaidou ${ }^{2}$, Diana Ferreira ${ }^{6}$, Miriam J. Johnson ${ }^{2}$ \\ and Magnus Ekström ${ }^{1,7}$
}

Affiliations: ${ }^{1}$ IMPACCT, Faculty of Health, University of Technology Sydney, Ultimo, Australia. ${ }^{2}$ Wolfson Palliative Care Research Centre, Hull York Medical School, University of Hull, Hull, UK. ${ }^{3}$ Silver Chain Group, Perth, Australia. ${ }^{4}$ Internal Medicine Dept, Ramathibodi Hospital, Mahidol University, Bangkok, Thailand. ${ }^{5}$ Marie Curie Palliative Care Research Group, Cardiff University, Cardiff, UK. ${ }^{6}$ Discipline, Palliative and Supportive Services, Flinders University, Adelaide, Australia. ${ }^{7}$ Dept of Clinical Sciences, Division of Respiratory Medicine and Allergology, Lund University, Lund, Sweden.

Correspondence: David C. Currow, Faculty of Health, University of Technology Sydney, PO Box 123, Ultimo, New South Wales 2007, Australia. E-mail: david.currowauts.edu.au

@ERSpublications

People with reasonable function and heart/lung diseases are at risk of breathlessness, increasing at the end of life http://ow.ly/nfNn30kZAUu

Cite this article as: Currow DC, Smith JM, Chansriwong P, et al. Missed opportunity? Worsening breathlessness as a harbinger of death: a cohort study. Eur Respir J 2018; 52: 1800684 [https://doi.org/ 10.1183/13993003.00684-2018].

ABSTRACT The aim of the study was to explore trajectories of breathlessness intensity by function and life-limiting illness diagnosis in the last 3 weeks of life in palliative care patients.

A prospective, consecutive cohort study obtained point-of-care data of patients of Silver Chain Hospice Care Service (Perth, Australia) over the period 2011-2014 ( $\mathrm{n}=6801 ; 51494$ data-points). Breathlessness intensity (0-10 numerical rating scale) and physical function (Australia-modified Karnofsky Performance Status (AKPS)) were measured at each visit. Time was anchored at death. Breathlessness trajectory was analysed by physical function and diagnosis using mixed effects regression.

Mean \pm SD age was $71.5 \pm 15.1$ years and $55.2 \%$ were male, most with cancer. The last recorded AKPS was $>40$ for $26.8 \%$. Breathlessness was worst in people with cardiorespiratory disease and AKPS $>40$, and breathlessness in the last week of life increased most in this group (adjusted mean 2.92 versus all others 1.51; $\mathrm{p}=0.0001)$. The only significant interaction was with diagnosis and function in the last week of life $(\mathrm{p}<0.0001)$.

Breathlessness is more intense and increases more in people with better function and cardiorespiratory disease immediately before death. Whether there are reversible causes for these people should be explored prospectively. Omitting function from previous population estimates may have overestimated breathlessness intensity for many patients in the days preceding death. 


\section{Introduction}

Chronic breathlessness affects one in 11 people in the general population [1]. For most people, this will be attributed to an underlying medical condition such as respiratory disease [2] which may be treatable, but not curable, and which is likely to contribute to suffering near death [3].

Severe breathlessness is one of the most frightening experiences. Unlike other symptoms such as pain, an episode of severe breathlessness is often associated with an ongoing terror of imminent death [4]. It is therefore unsurprising that the mode of dying, in particular a fear of severe breathlessness or suffocation, may be a major anxiety for people with a life-limiting illness [5]. Many people may have vivid memories of a relative dying with serious respiratory distress, and past memories and fears affect central perception and anticipation of breathlessness [6].

In the weeks prior to death in a palliative care population, the prevalence and intensity of breathlessness is reported to increase and functional status most often worsens $[7,8]$. This phenomenon has been described in several datasets and includes people with a wide range of recorded causes of death, including frailty, dementia and those perceived to have had a "sudden" death [9-12]. To date, two cohort studies have proposed contradictory effects of performance status on chronic breathlessness at the end of life: one suggested that decreasing performance status is associated with the increasing breathlessness and the other, the opposite $[10,13]$. Given that no study has evaluated whether the trajectory of breathlessness differs in relation to the patient's physical functional status and underlying condition(s) in the last weeks before death, and the role of functional status in the two studies that have been done to date was contradictory, this current study was conceived to explore this relationship.

The relationship between breathlessness and functional status at the end of life is important to inform clinical discussions with concerned patients and their relatives, and to guide research in ways of best palliating breathlessness. At least two distinct trajectories of function and their relation to breathlessness need to be understood: 1) progressive functional decline and 2) sudden deterioration in the setting of a known life-limiting illness $[9,12]$. Such patterns may also provide important insights into the underlying pathologies leading to different trajectories of dying and the need to pay heed to symptom exacerbations even in the last weeks of life.

The aim of this study was to evaluate patterns of breathlessness by levels of function in the last 3 weeks of life in palliative care. The null hypothesis was that there would be no difference in the trajectories of breathlessness intensity between diagnostic groups categorised by function in the 21 days preceding death.

\section{Methods}

Study setting

Silver Chain Hospice Care Service (SCHCS) is the sole community palliative care provider for metropolitan Perth in Australia, providing care for approximately 2800 patients annually in their own homes. The SCHCS comprises an interdisciplinary team that includes general practitioners, registered nurses, care aides, volunteers, counsellors and pastoral care workers. Registered nurses are available $24 \mathrm{~h}$ per day, 7 days per week, supported by clinical nurse consultants and other staff. The service is funded by the state government and is free of charge to palliative care patients.

\section{Study cohort and design}

This was a longitudinal study using all data on breathlessness and level of function, aggregated each day from a consecutive cohort of 7896 patients seen by the SCHCS between January 1, 2011 and December 31, 2014. There were 1095 patients who had no data recorded in the last 21 days of life. These patients were excluded, leaving 6801 patients for analysis with 51494 data-points [7, 14, 15]. We included all data-points collected in the last 21 days of life for everyone who died, with simultaneously recorded breathlessness intensity and functional scores, categorised into broad diagnostic groups. As the only time-point that is constant in referral-dependent hospice and palliative care is death [16], the longitudinal analysis used time anchored at death in this cohort of people whose expected outcome was death.

\section{Data collection from SCHCS records}

All data were collected in the clinical setting contemporaneously with each face-to-face visit by a health professional from the SCHCS. Processes including staff recording of patient rating of symptoms remained constant during the study period. De-identified data included demographic and clinical data recorded once on admission to the service, and clinical data recorded at each clinical encounter. Data quality was optimised by routine point-of-care data entry by SCHCS staff into mobile phone data systems for all fields relating to that clinical encounter. 
Demographic and clinical data recorded once on admission to the service

1) Demographic characteristics (age, sex, living arrangement, caregiver status and place of death). 2) Dominant cause of life-limiting illness (cardiorespiratory diseases (primary lung cancer, respiratory disease, cardiac failure) and other).

\section{Clinical data recorded at each clinical encounter}

1) Patient-rated intensity of breathlessness on an 11-point numerical rating scale (NRS) between 0 ("no") to 10 ("worst imaginable") using the Symptom Assessment Scale [17]. 2) Clinician-rated performance status using the Australia-modified Karnofsky Performance Status (AKPS) scale ranging from 100 ("functioning normally and without symptoms or assistance") to 0 ("dead") in 10-point decrements. An AKPS of 40 indicates high levels of care for basic activities of daily living and a score of 20 represents almost total dependence on others for care. AKPS has been validated in palliative care populations [18]. 3) Patients were visited in their homes by clinicians as required. This may have been on a weekly basis, every few days, daily or several times in a day. Frequency of visits tend to increase as time of death approaches, but depends on the needs of the patients and their families.

\section{Statistical analyses}

The cohort and service characteristics were reported using descriptive statistics, and were compared using Chi-squared analyses for categorical data, ANOVA for continuous variables for normally distributed data and the Kruskal-Wallis test for nonnormally distributed data. Data were tested for normality by graphing them.

For each of the last 21 days of life, the intensity of breathlessness was compared between people with an AKPS $\leqslant 40$ on that day and those with higher functional status, and between people with underlying cardiorespiratory disease and those with other diseases. The secondary comparators were the trajectories of

TABLE 1 Characteristics of a consecutive cohort of people referred to a community palliative care service between January 2011 and December 2014 including diagnostic group, and breathlessness and functional scores

\begin{tabular}{|c|c|c|c|}
\hline & $\begin{array}{c}\text { No } \\
\text { cardiorespiratory } \\
\text { disease }\end{array}$ & $\begin{array}{c}\text { Cardiorespiratory } \\
\text { disease }\end{array}$ & Total \\
\hline Subjects $n$ & 5114 & 1687 & 6801 \\
\hline \multicolumn{4}{|l|}{$\operatorname{Sex} n(\%)$} \\
\hline Male & $2734(53.46)$ & $1017(60.28)$ & $3751(55.2)$ \\
\hline Female & $2380(46.54)$ & $670(39.72)$ & $3050(44.8)$ \\
\hline Age at death years mean $\pm s D$ & $71.0 \pm 16.0$ & $72.7 \pm 11.7^{\#}$ & $71.5 \pm 15.1$ \\
\hline \multicolumn{4}{|l|}{ Diagnosis $\mathrm{n}(\%)$} \\
\hline \multicolumn{4}{|l|}{ Cardiorespiratory disease } \\
\hline Primary lung cancer & & & $1398(20.6)$ \\
\hline Respiratory failure & & & $153(2.3)$ \\
\hline Cardiac failure & & & $136(2.00)$ \\
\hline No documented cardiorespiratory disease & & & $5114(75.2)$ \\
\hline Time from referral to death days median (IQR) & 40 (78) & $51(84)^{\pi}$ & $42(79)$ \\
\hline $\begin{array}{l}\text { Observations per patient in the last week of life } \\
\text { median (IQR) }\end{array}$ & $7(6)$ & $6(5)$ & $7(6)$ \\
\hline \multicolumn{4}{|l|}{ Last measurement before death } \\
\hline \multicolumn{4}{|l|}{ AKPS n (\%) } \\
\hline$>40$ & 544 (32.2) & $1282(25.1)$ & $1826(26.8)$ \\
\hline$\leqslant 40$ & $3831(74.9)$ & $1143(67.7)$ & $4974(73.2)$ \\
\hline \multicolumn{4}{|l|}{ Days median } \\
\hline AKPS $>40$ & 8 & 7 & 1 \\
\hline AKPS $\leqslant 40$ & 0 & 0 & \\
\hline Breathlessness (0-10 NRS) median (IQR) & $0(4)$ & $3(4)^{+}$ & $1(4)$ \\
\hline \multicolumn{4}{|c|}{ 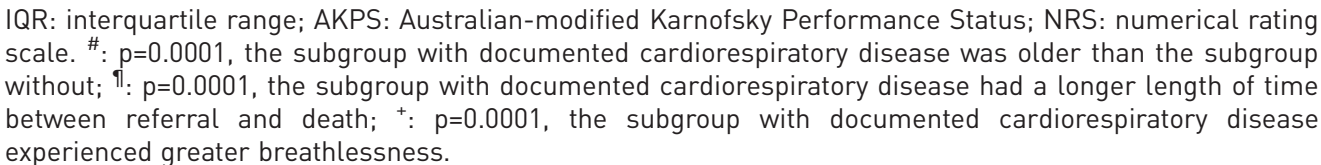 } \\
\hline
\end{tabular}


breathlessness by 10-point AKPS $(10,20,30$ or 40) in the last 3 weeks of life, further stratified by the presence of cardiorespiratory disease. Lung cancer was included in the cardiorespiratory group.

The number of days from death was transformed into a three-point categorical variable with the last 7 days of data being included into week 1, week 2 including 8-14 days and week 3 including 15-21 days from death.

Factors related to change in breathlessness were analysed using mixed effects regression accounting for repeated measurements, adjusted for age, sex, carer status, length of stay (from referral to the service until death) and diagnostic group. Interaction terms between week from death and 1) AKPS, 2) cardiorespiratory diagnosis, and 3) both AKPS and cardiorespiratory diagnosis were evaluated by including interaction terms in the fully adjusted model. Mean differences in breathlessness intensity were presented with $95 \%$ confidence intervals. No missing data were imputed. Statistical significance was defined as a two-sided $\mathrm{p}<0.05$. Data were analysed using Stata version 14.0 (StataCorp, College Station, TX, USA).

\section{Ethical considerations}

The study was approved by Silver Chain Human Research Ethics Committee. Given that these were aggregated, de-identified data, individual consent was not required. This paper is reported using the STROBE (Strengthening the Reporting of Observational Studies in Epidemiology) guidelines [19].

\section{Results}

In total, 6801 people were included in the analysis, generating 51494 data-points in the last 21 days of life; $55.2 \%$ males, mean \pm SD age at death $71.5 \pm 15.1$ years and mean \pm SD time from referral to death 78.4 \pm 107.1 days (table 1 ). Most people had cancers as their documented life-limiting illness (5114 out of 6801 ; $75.2 \%$ ). Of the people who died, $26.8 \%$ (1826 out of 6801 ) had their last recorded AKPS $>40$. Similar numbers of assessments were carried out in the last week of life in those with and without cardiorespiratory disease, and the time from the last recorded clinical observation until death for each subpopulation was similar (table 1).

Breathlessness was higher in people with cardiorespiratory disease at all time-points. Breathlessness was also higher in people with better levels of function, and increased in intensity most markedly in people with cardiorespiratory disease and higher levels of function (figure 1). People with a cardiorespiratory life-limiting illness also had higher breathlessness intensity scores (adjusted mean 2.92 on a 0-10 NRS) than people with no documented cardiorespiratory diagnosis on each day (adjusted mean of 1.51; $\mathrm{p}=0.0001)$. This was seen in both AKPS groups $(\mathrm{p}<0.001$ for each). This was seen in the AKPS strata

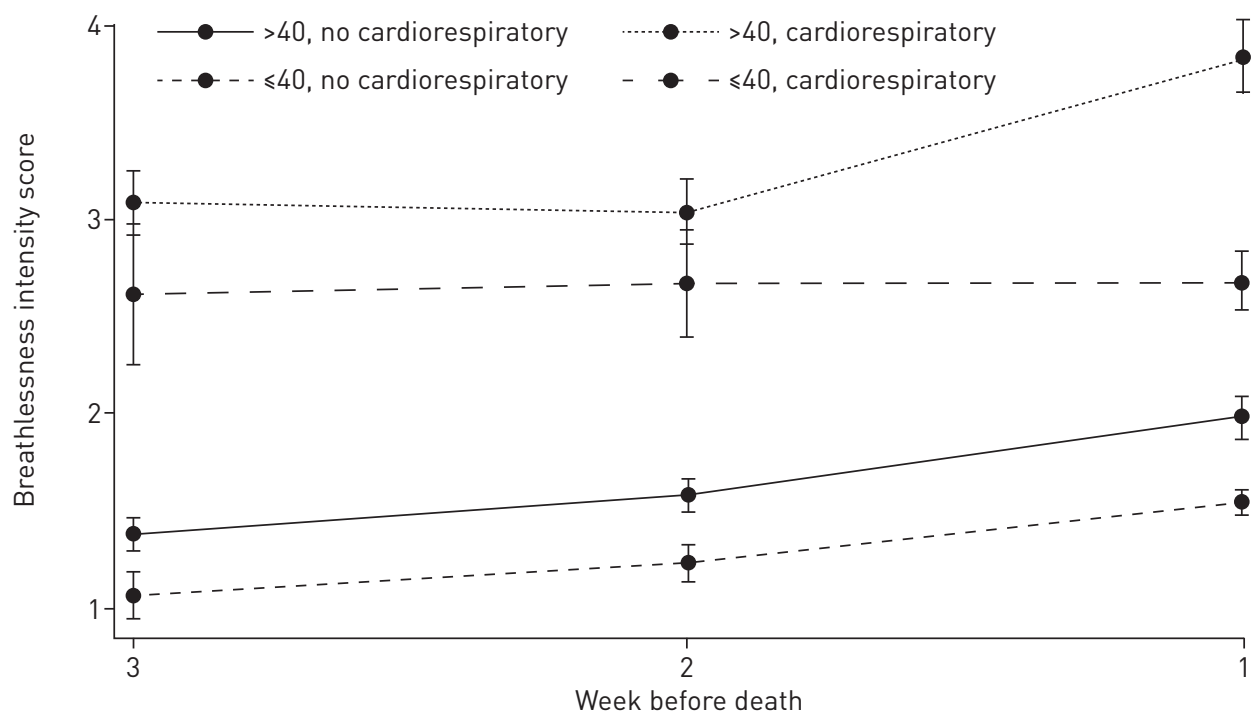

FIGURE 1 Adjusted mean breathlessness intensity scores on a 0-10 numerical rating scale in the 3 weeks before death by diagnosis (cardiorespiratory life-limiting illness or not) and functional status ( $\leqslant 40$ or $>40$ using the Australia-modified Karnofsky Performance Status (AKPS) scale) having adjusted for age, sex and length of stay. 6768 clients with 48385 observations. People with a cardiorespiratory life-limiting illness had higher breathlessness intensity scores ladjusted mean 2.92) than people with no documented cardiorespiratory diagnosis on each day ladjusted mean 1.51; $p=0.0001)$. This was seen in both AKPS groups $(p<0.001$ for each). 
dichotomised between 40 and 50 by diagnostic group ( $p<0.001$ for each) (figure 1$)$. These differences were clinically significant [20] (table 2). When split by cardiorespiratory status and AKPS 10, 20, 30 and $\geqslant 40$, similar patterns were seen (supplementary figure S1).

In the adjusted mixed effects regression model accounting for repeated measures and adjusting for the available factors, independent predictors of worsening breathlessness were proximity to death $(0.6,95 \%$ CI 0.49-0.72), cardiorespiratory disease (breathlessness was 1.72 points worse, 95\% CI $1.53-1.90$ points worse), while factors reducing the likelihood of breathlessness included poorer performance status $(0.31$, 95\% CI $0.17-0.45)$ and being female $(0.28,95 \%$ CI $0.18-0.37)$. The relationship between breathlessness and a person's level of function remained significant in the mixed effects regression model when interaction terms (week $\times$ diagnosis; week $\times$ function; week $\times$ diagnosis $\times$ function) were added, with the only significant interaction term being diagnosis and function in the last week of life $(p<0.0001)$, suggesting that in the last week, there is a real difference in trajectories between groups.

\section{Discussion}

This is the first study to stratify trajectories of breathlessness by functional status near death and whether the person has a cardiorespiratory life-limiting illness, extending previous analyses of breathlessness in the final weeks of life $[7,13]$. The increase in breathlessness in the last 21 days of life is seen least in the numerically largest of four subgroups in the data, i.e. those with poorer levels of function and no documented cardiorespiratory disease. This group did not experience the same crescendo in breathlessness seen in the subgroup with better function and cardiorespiratory disease. Numerically, the smallest subgroup (i.e. those with better function and cardiorespiratory life-limiting illness) had the highest levels of breathlessness intensity across the last 21 days of life and experienced the largest absolute increase in breathlessness across that time.

Previous studies have reported that breathlessness increases as death approaches (along with fatigue), whereas other symptoms tend to decrease $[7,10,13]$. Importantly, in contrast to this new analysis, none of these studies adjusted for functional status. These three previous analyses adjusted for underlying life-limiting illnesses and defined higher levels of breathlessness in people with chronic respiratory disease. Bringing both factors into the current analysis sees the increase in breathlessness as death approaches disappear for the majority of patients, suggesting that the results of previous reports were being driven by increases seen in the breathlessness scores before their sudden death from this one subgroup, i.e. the

TABLE 2 Multivariate model of breathlessness in relation to time before death, diagnosis and function adjusted for confounders

Coefficient $(95 \% \mathrm{Cl})$

p-value $>|z|$

\begin{tabular}{|c|c|c|}
\hline \multicolumn{3}{|l|}{ Breathlessness } \\
\hline \multicolumn{3}{|l|}{ Week 3} \\
\hline Week 2 & $0.203(0.115-0.291)$ & $<0.001$ \\
\hline Week 1 & $0.607(0.491-0.724)$ & $<0.001$ \\
\hline Age & $0.001(-0.002-0.005)$ & 0.401 \\
\hline Length of stay & $0.000(-0.000-0.001)$ & 0.240 \\
\hline \multicolumn{3}{|l|}{ Diagnosis } \\
\hline \multicolumn{3}{|l|}{ No cardiorespiratory } \\
\hline Cardiorespiratory & $1.716(1.529-1.904)$ & $<0.001$ \\
\hline \multicolumn{3}{|l|}{ AKPS } \\
\hline \multicolumn{3}{|l|}{$>40$} \\
\hline$\leqslant 40$ & $-0.314(-0.453--0.175)$ & $<0.001$ \\
\hline \multicolumn{3}{|c|}{ - } \\
\hline \multicolumn{3}{|l|}{ Male } \\
\hline Female & $-0.275(-0.371--0.179)$ & $<0.001$ \\
\hline \multicolumn{3}{|c|}{$\begin{array}{l}\text { Interaction between week, functional status and } \\
\text { cardiorespiratory diagnoses }\end{array}$} \\
\hline Week $3, \leqslant 40$, cardiorespiratory & $-0.147(-0.547-0.252)$ & 0.470 \\
\hline Week $2, \leqslant 40$, cardiorespiratory & $-0.015(-0.329-0.299)$ & 0.926 \\
\hline Week $1, \leqslant 40$, cardiorespiratory & $-0.711(-0.956--0.466)$ & $<0.001$ \\
\hline
\end{tabular}


people with higher levels of function and documented cardiorespiratory disease. Previous data may therefore have been skewed across the population in the weeks leading up to death by the data from this new identifiable subgroup.

Chronic heart failure has a trajectory of acute exacerbations interspersed with periods of stability [21]. Recognition of worsening symptoms may allow intense support and recovery nearly to previous levels of function. In heart failure, as the disease severity worsens, death from progressive circulatory failure becomes relatively more common compared with sudden arrhythmic death [22]. This means that the predicted mode of death (sudden versus nonsudden) may change with advancing symptoms and signs of disease, and this helps better target interventions. For example, cardiac defibrillators are not recommended for patients with New York Heart Association class IV disease [23]. During these acute exacerbations or as a result of arrhythmias or infarction, a group of people with reasonable function died very rapidly.

Apparent "sudden" cardiac death may have premonitory symptoms, most commonly chest pain or breathlessness, even in younger, apparently fit people, or may be secondary to another terminal event [24, 25]. A cohort study of middle-aged people suffering out-of-hospital sudden cardiac arrest found that some had had symptoms not just in the preceding $24 \mathrm{~h}$, but over the previous 4 weeks [26]. For this reason, information on events immediately surrounding death and in the weeks leading up to death will help in the future to better understand the disease trajectory and identify any opportunities to modify clinical care.

The worsening levels of breathlessness may represent events which could be treatable or, in the case of pulmonary emboli, preventable. Pulmonary emboli are reported in $50 \%$ of autopsies in patients with cancer yet it is unclear whether these represent the sole cause of death, a contributory pathology within the agonal process $[27,28]$ or a clinically incidental finding. Since $50 \%$ of hospice inpatients have evidence of asymptomatic deep vein thrombosis when sought [29], and hospice inpatients with a higher risk of venous thromboembolism (VTE) are more likely to have symptoms known to be related to VTE [30], it is reasonable to consider VTE as a potential contributory factor to worsening breathlessness in those who die from inanition and those with a higher AKPS who experience a "sudden death". Indeed, it has been demonstrated that fatal, apparently sudden, pulmonary emboli are rarely asymptomatic, and are dominated by tachycardia and breathlessness often with symptoms preceding death by days or weeks having been either ignored or misattributed [31]. People with incidentally diagnosed cancer-related VTE are often found on closer questioning to have had symptoms for some time [32, 33].

\section{Mechanisms}

The present findings confirm an increase in breathlessness intensity as death approaches, but the increase is more marked in people with cardiorespiratory disease and, then, those with better function. In this latter group, it is not apparent whether breathlessness intensity is a result of continued mobility, acute intercurrent pathology, faster progression of the person's primary condition or a mixture of all three. Acute pathology manifesting itself over several weeks is a likely contributor for many people given the magnitude of increase seen.

People with lung cancer form a substantial proportion of the group with cardiorespiratory disease and higher levels of function. By contrast, in the subgroup of people with better levels of function and no documented cardiorespiratory disease (composed almost entirely of people with cancer), the pattern of markedly worsening breathlessness is not seen in the week before death, raising the possibility that VTE with a prodrome of breathlessness is less likely to be a contributing factor to sudden death. Such hypotheses can only be tested in a bespoke, prospective, longitudinal study.

\section{Strengths and limitations}

This was a large, prospectively collected dataset used in clinical care, with low levels of missing data and an emphasis on patient-reported measures during clinical encounters. It has a large number of observations for each patient admitted to the service. The numbers of observations increase each week as death approaches.

As this study does not include people admitted to hospital for their terminal care, the overall levels of breathlessness intensity may be underestimated as people with conditions causing increasing breathlessness (e.g. acute coronary syndrome, arrhythmias, pulmonary embolism and acute lower respiratory tract infections) may be more likely to be admitted. Furthermore, breathlessness as a reason for admission to hospital was not available either from this dataset.

There are no data distinguishing between patient-reported measures and proxy measures. The latter are more likely to be needed in people with very poor levels of AKPS (10 or 20) and may underestimate the intensity of breathlessness, although some studies have found family and specialist palliative care staff proxy assessments are reasonably valid for breathlessness [34-37]. Whether breathlessness was occurring 
at rest, on minimal exertion or, in the case of people with better functional status, on even greater levels of exertion cannot be derived from the available data.

Data on cause of death were not available and a clinical review of several thousand cases was beyond the scope of this current project. Likewise, given the documented inaccuracy of death certificates, these were not sought for this study [38].

A priori, the clinical categories were defined. Given the high correlation between prolonged exposure to tobacco, chronic obstructive pulmonary disease and lung cancer, it was decided to include primary (but not secondary) lung cancer in the group of patients with cardiorespiratory diseases.

\section{Implications for clinical practice}

In people with metastatic cancer, the cut-point of AKPS used in this study has prognostic significance. In one consecutive cohort, median survival in people with AKPS $\leqslant 40$ was 29 days compared with 146 days for those with AKPS $>40$ and poor physical wellbeing [39]. The sudden deaths described in this current analysis may therefore be a clinically important decrement in life expectancy, especially if potentially reversible causes can be identified. This may be of great importance to many patients.

There appears to be a warning of increasing breathlessness 7-9 days before death; for some patients, this is likely to allow for potentially reversible causes to be investigated and treated, if that were the patient's wish, or to support the patient and family in their preferred place of death. This study suggests that worsening breathlessness should alert clinicians to an increased likelihood of death in people with reasonable function in the setting of documented cardiorespiratory disease. For people with better functional status, this raises the question of whether there are reversible causes for both the symptom and for what may be a premature death in these circumstances. There appears to be a window for a clinical response to define potentially reversible factors.

In addition, this study is a reminder that some causes of sudden death may be preventable. The incidence of VTE increases with cancer progression and it remains the second most common cause of death associated with malignancy $[27,28,40]$. Despite national policies to implement VTE risk assessment and thromboprophylaxis of all people admitted to hospital, this has very low take-up in palliative care units [41]. Here, people with AKPS $>40$ may well be admitted for symptom management rather than end-of-life care, yet risk assessment for and thromboprophylaxis of VTE is not standard practice [41, 42]. Hospice and palliative care services should review their approach to risk assessment for VTE and thromboprophylaxis for those at higher risk.

\section{Implications for future research}

Future research should focus on whether any increase in breathlessness in people with a progressive, life-limiting disease has an underlying and potentially modifiable cause such as VTE, cardiac ischaemia, arrhythmia or infection. For example, previous work has suggested systematic underestimation of deep vein thromboses in this patient population [43].

The two trajectories that are identified in this study need to be documented with more clinical detail in a large, prospective, consecutive cohort study designed specifically to analyse these current findings. The first trajectory hypothesised is for people with an AKPS $\leqslant 40$ who appear to have an inexorable decline associated with lower levels of breathlessness and are more likely to be experiencing progressive cachexia. The second trajectory hypothesised may represent the relatively unexpected death of patients with better functional status preceding death and much higher levels of breathlessness. Acute events including infections, cardiac ischaemia, arrhythmias, pulmonary emboli or pericardial effusions, any of which may cause relatively acute worsening of breathlessness intensity in people with pre-existing cardiorespiratory disease, could help to explain the findings in this current study. If breathlessness in people with relatively good function and documented cardiorespiratory disease is a harbinger of impending death, this has significant clinical implications.

There will also need to be a larger cohort that includes inpatients and people who have presented to the emergency department to have a fuller picture of changes in breathlessness intensity by level of function at the end of life.

Conflict of interest: D.C. Currow received an unrestricted research grant from Mundipharma, is an unpaid member of an advisory board for Helsinn Pharmaceuticals, has been a consultant to Specialist Therapeutics and Mayne Pharma, and received intellectual property payments from Mayne Pharma.

Support statement: This study drew on discretionary funding held by the researchers and staff positions. No external, competitive funding contributed to this study. 


\section{References}

1 Currow DC, Plummer J, Crockett A, et al. A community population survey of prevalence and severity of dyspnoea in adults. J Pain Symptom Manage 2009; 38: 533-545.

2 Johnson M, Bowden J, Abernethy AP, et al. To what causes do people attribute their chronic breathlessness? A population survey. J Palliat Med 2012; 15: 744-750.

3 Currow DC, Clark K, Kamal A, et al. The population burden of chronic symptoms that substantially predate the diagnosis of a life-limiting illness. J Palliat Med 2015; 18: 480-485.

4 Bailey PH. Death stories: acute exacerbations of chronic obstructive pulmonary disease. Qual Health Res 2001; 11: 322-338.

5 Gysels MH, Higginson IJ. The lived experience of breathlessness and its implications for care: a qualitative comparison in cancer, COPD, heart failure and MND. BMC Palliat Care 2011; 10: 15

6 Herigstad M, Hayen A, Wiech K, et al. Dyspnoea and the brain. Respir Med 2011; 105: 809-817.

7 Currow DC, Smith J, Davidson PM, et al. Do the trajectories of dyspnoea differ in prevalence and intensity by diagnosis at the end of life? A consecutive cohort study. J Pain Symptom Manage 2010; 39: 680-690.

8 Johnson MJ, Bland JM, Gahbauer E, et al. Breathlessness in elderly adults during the last year of life sufficient to restrict activity. Prevalence, pattern, and associated factors. J Am Geriatr Soc 2016; 64: 73-80.

9 Ekström M, Vergo MT, Ahmadi Z, et al. Prevalence of sudden death in palliative care: data from the Australian Palliative Care Outcomes Collaborative. J Pain Symptom Manage 2016; 52: 221-227.

10 Ekström M, Johnson MJ, Kaasa S, et al. Who experiences higher and increasing breathlessness in advanced cancer? The longitudinal EPCCS Study. Support Care Cancer 2016; 24: 3803-3811.

11 Seow H, Barbera L, Sutradhar R, et al. Trajectory of performance status and symptom scores for patients with cancer during the last six months of life. J Clin Oncol 2011; 29: 1151-1158.

12 Currow DC, Vergo ML, Ekstrom M. Sudden death in palliative care. J Pain Symptom Manage 2015; 50: e1-e2.

13 Ekström PM, Allingham S, Eagar K, et al. Breathlessness during the last week of life in palliative care: an Australian prospective, longitudinal study. J Pain Symptom Manage 2016; 51: 816-823.

14 Currow DC, Agar M, Smith J, et al. Does palliative home oxygen improve dyspnoea? A consecutive cohort study. Palliat Med 2009; 23: 309-316.

15 Currow DC, Christou T, Smith J, et al. Do terminally ill people who live alone miss out on home oxygen treatment? An hypothesis generating study? J Palliat Med 2008; 11: 1015-1022.

16 Currow DC, Abernethy AP, Fazekas BS. Specialist palliative care needs of whole populations. A feasibility study using a novel approach. Palliat Med 2004; 18: 239-247.

17 Kristjanson L, Pickstock S, Yuen K, et al. Development and Testing of the Revised Symptom Assessment Scale (Final Report). Perth, Edith Cowan University, 1999.

18 Abernethy AP, Shelby-James T, Fazekas BS, et al. The Australia-modified Karnofsky Performance Status (AKPS) scale: a revised scale for contemporary palliative care clinical practice [ISRCTN81117481]. BMC Palliat Care 2005; 4: 7.

19 Von Elm E, Altman DG, Egger M, et al. The Strengthening the Reporting of Observational Studies in Epidemiology (STROBE) statement: guidelines for reporting observational studies. Prev Med 2007; 45: 247-251.

20 Johnson MJ, Bland JM, Oxberry SG, et al. Clinically important differences in the intensity of chronic refractory breathlessness. J Pain Symptom Manage 2013; 46: 957-963.

21 Goodlin SJ, Hauptman PJ, Arnold R, et al. Consensus statement: palliative and supportive care in advanced heart failure. J Card Fail 2004; 10: 200-209.

22 MERIT-HF Study Group. Effect of metoprolol CR/XL in chronic heart failure: Metoprolol CR/XL Randomised Intervention Trial in Congestive Heart Failure (MERIT-HF). Lancet 1999; 353: 2001-2007.

23 National Institute for Health and Care Excellence. Implantable cardioverter defibrillators and cardiac resynchronisation therapy for arrhythmias and heart failure. 2014. www.nice.org.uk/guidance/ta314/chapter/ 1-guidance Date last accessed: November 16, 2017.

24 Pratt CM, Greenway PS, Schoenfeld MH, et al. Exploration of the precision of classifying sudden cardiac death. Implications for the interpretation of clinical trials. Circulation 1996; 93: 519-524.

25 Kinch Westerdahl A, Sjöblom J, Mattiasson AC, et al. Implantable cardioverter-defibrillator therapy before death: high risk for painful shocks at end of life. Circulation 2014; 129: 422-429.

26 Marijon E, Uy-Evanado A, Dumas F, et al. Warning symptoms are associated with survival from sudden cardiac arrest. Ann Intern Med 2016; 164: 23-29.

27 Sproul EE. Carcinoma and venous thrombosis: the frequency of association of carcinoma in the body or tail of the pancreas with multiple venous thrombosis. Am J Cancer 1938; 34: 566-585.

28 Ambrus JL, Ambrus CM, Mink IB, et al. Causes of death in cancer patients. J Med 1975; 6: 61-64.

29 Johnson MJ, Sproule MW, Paul J. The prevalence and associated variables of deep venous thrombosis in patients with advanced cancer? Prevalence and associated variables. Clin Oncol 1999; 11: 105-110.

30 Johnson MJ, McMillan B, Fairhurst C, et al. Primary thromboprophylaxis in hospices: the association between risk of venous thromboembolism and development of symptoms. J Pain Symptom Manage 2014; 48: 56-64.

31 Havig O. Deep vein thrombosis and pulmonary embolism. An autopsy study with multiple regression analysis of possible risk factors. Acta Chir Scand Suppl 1977; 478: 1-120.

32 Cronin CG, Lohan DG, Keane M, et al. Prevalence and significance of asymptomatic venous thromboembolic disease found on oncologic staging CT. AJR Am J Roentgenol 2007; 189: 162-170.

33 van Es N, Bleker SM, Di Nisio M. Cancer-associated unsuspected pulmonary embolism. Thromb Res 2014; 133 Suppl. 2, S172-S178.

34 Higginson I, Priest P, McCarthy M. Are bereaved family members a valid proxy for a patient's assessment of dying? Soc Sci Med 1994; 38: 553-557.

35 Quinn C, Dunbar SB, Higgins M. Heart failure symptom assessment and management: can caregivers serve as proxy? J Cardiovasc Nurs 2010; 25: 142-148.

36 Moody JE, McMillan S. Dyspnea and quality of life indicators in hospice patients and their caregivers. Health Qual Life Outcomes 2003; 1: 9 .

37 Simon ST, Altfelder N, Alt-epping B, et al. Is breathlessness what the professional says it is? Analysis of patient and professionals' assessments from a German nationwide register. Support Care Cancer 2014; 22: 1825-1832. 
38 Johansson LA, Westerling R. Certificates: implications for mortality statistics. Int J Epidemiol 2000; 29: 495-502.

39 Hwang SS, Scott CB, Chang VT, et al. Prediction of survival for advanced cancer patients by recursive partitioning analysis: role of Karnofsky performance status, quality of life, and symptom distress. Cancer Invest 2004; 22: $678-687$.

40 Blom JW, Doggen CJ, Osanto S, et al. Malignancies, prothrombotic mutations, and the risk of venous thrombosis. JAMA 2005; 293: 715-722.

41 ISTH Steering Committee for World Thrombosis Day. Venous thromboembolism: a call for risk assessment in all hospitalised patients. Thromb Haemost 2016; 116: 777-779.

42 Noble S, Finlay IG. Have Palliative Care Teams' attitudes to venous thromboembolism changed? A survey of thromboprophylaxis practice across British Specialist Palliative Care Units in the years 2000 and 2005 . J Pain Symptom Manage 2006; 32: 38-43.

43 Noble S, Nelson A, Finlay IG. Factors influencing hospice thromboprophylaxis policy: a qualitative study. Palliat Med 2008; 22: 808-813. 